\title{
The pion form factor from analyticity and unitarity
}

\author{
B ANANTHANARAYAN ${ }^{1}$, IRINEL CAPRINI ${ }^{2}$ \\ and I SENTITEMSU IMSONG ${ }^{1, *}$ \\ ${ }^{1}$ Centre for High Energy Physics, Indian Institute of Science, Bangalore 560012 , India \\ ${ }^{2}$ Horia Hulubei National Institute for Physics and Nuclear Engineering, P.O.B. MG-6, \\ 077125 Magurele, Romania \\ *Corresponding author. E-mail: senti@cts.iisc.ernet.in
}

\begin{abstract}
Analyticity and unitarity techniques are employed to estimate Taylor coefficients of the pion electromagnetic form factor at $t=0$ by exploiting the recently evaluated two-pion contribution to the muon $(g-2)$ and the phase of the pion electromagnetic form factor in the elastic region, known from $\pi \pi$ scattering by Fermi-Watson theorem and the values of the form factor at several points in the space-like region. Regions in the complex $t$-plane are isolated where the form factor cannot have zeros.
\end{abstract}

Keywords. Unitarity; analyticity; zeros.

PACS Nos 11.30.Rd; 13.40.Gp

\section{Introduction}

The pion electromagnetic form factor $F_{\pi}(t)$ is an important object to account for a variety of phenomena in strong interaction dynamics. $F_{\pi}(t)$ is a real analytic function in the $t$-plane cut along the real axis from the unitarity threshold $t_{+}=4 M_{\pi}^{2}$ to $\infty$ and is normalized to $F_{\pi}(0)=1$. The values of $F_{\pi}(t)$ have been measured recently with increasing precision at space-like values $Q^{2}>0$ [1]. On the time-like cut, where the form factor is complex, the Fermi-Watson theorem implies that in the elastic region, its phase is equal to the phase-shift of the $P$-wave of the $\pi \pi$ amplitude, calculated recently with precision using Roy equations and fixed- $t$ dispersion relations. On the other hand, the modulus has been measured from the cross-section of $e^{+} e^{-} \rightarrow \pi^{+} \pi^{-}$to high accuracy by the BABAR and KLOE Collaborations (for references, see ref. [2]). These data have been used for an accurate evaluation of the two-pion contribution to the muon anomalous magnetic moment [3] which is the input for our work. The method requires the Omnès function for the phase from $4 m_{\pi}^{2} \leq t \leq t_{\text {in }}$. In the present paper, we find constraints on the coefficients $c$ and $d$ appearing in the Taylor expansion:

$$
F(t)=1+\frac{1}{6}\left\langle r_{\pi}^{2}\right\rangle t+c t^{2}+d t^{3}+\cdots
$$


using a technique discussed in ref. [2], which makes use of information on both the phase and modulus and the value of the form factor in the space-like region. We also consider the problem of the zeros, and obtain regions in the complex $t$-plane where zeros are excluded.

\section{Review of the method}

We include information on the modulus via an integral relation

$$
\frac{1}{\pi} \int_{t_{\text {in }}}^{\infty} \mathrm{d} t \rho(t)|F(t)|^{2} \leq I,
$$

where $\rho(t)$ is a positive definite weight in the region of integration and $I$ is a known quantity. We make the particular choice for $I$ that corresponds to the two-pion contribution to the $(g-2)$ of the muon. We transform our problem via a conformal map, cast the integral equation into a canonical form and derive a determinant (for details, see ref. [2]) which is central to our investigations for obtaining bounds on the low-energy Taylor series expansion coefficients of $F_{\pi}$ and for finding regions of excluded zeros in the complex $t$-plane.

\section{Inputs and results}

A detailed description for the phase, modulus and the space-like data used have been presented in Sec. III and in Tables I and II of ref. [2]. We take $\sqrt{t_{\text {in }}}=0.917 \mathrm{GeV}$, which corresponds to the first important inelastic threshold due to the $\omega \pi$ pair. A very precise parametrization of the phase-shift $\delta_{1}^{1}$ which we have used in our work is given in ref. [4].

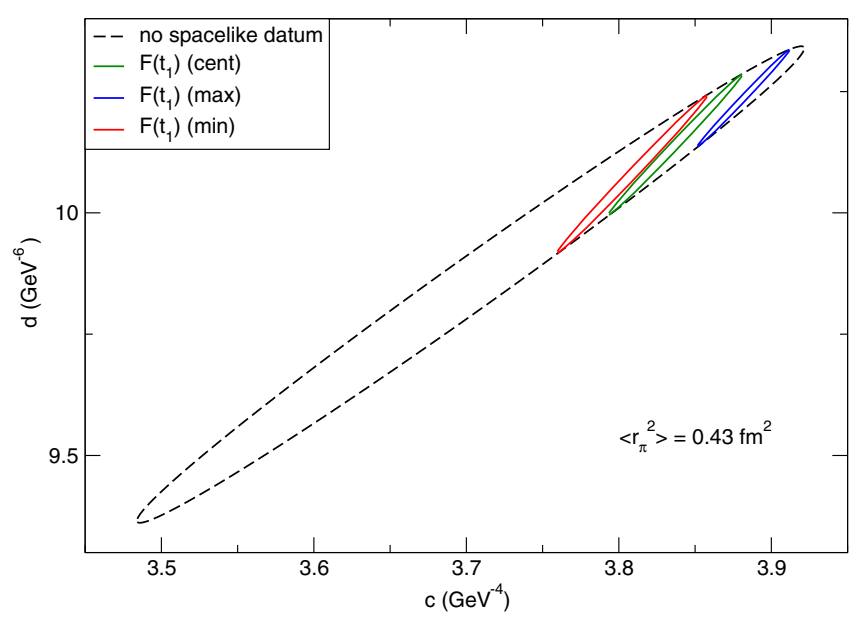

Figure 1. Allowed domain in the $c-d$ plane calculated with $t_{\text {in }}=(0.917 \mathrm{GeV})^{2}$ and $\left\langle r_{\pi}^{2}\right\rangle=0.43 \mathrm{fm}^{2}$ for three values of $F\left(t_{1}\right)$ (central value in Table VII of ref. [1] and the extreme values obtained from the error intervals) at the space-like point $t_{1}=-1.6 \mathrm{GeV}^{2}$. Also shown is the bigger ellipse with just phase and modulus. 


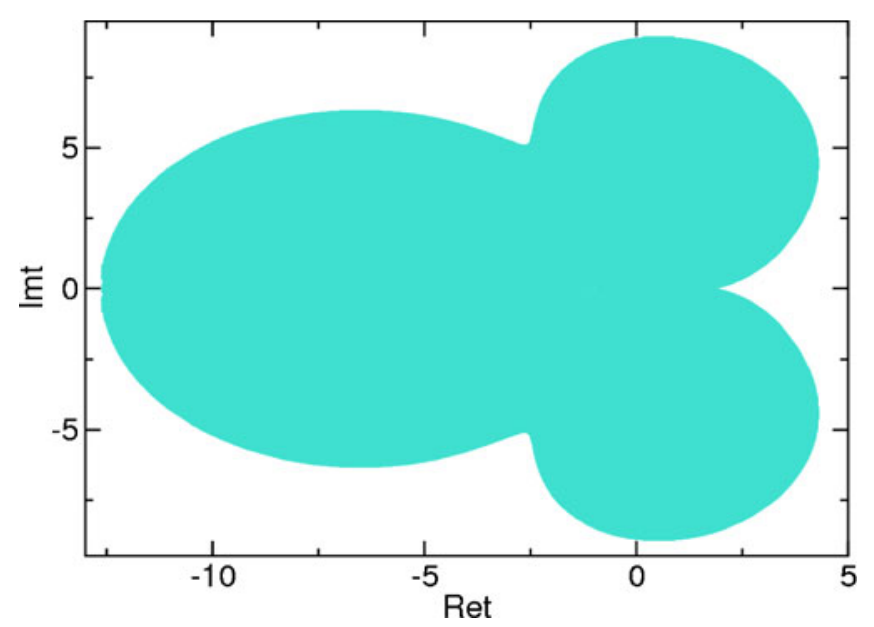

Figure 2. Domain without zeros obtained with $t_{\text {in }}=(0.917 \mathrm{GeV})^{2}$ and $\left\langle r_{\pi}^{2}\right\rangle=$ $0.43 \mathrm{fm}^{2}$, using in addition, the central experimental value $F\left(t_{1}\right)=0.243$ (the central experimental value given in Table VII of ref. [1]) at the space-like point $t_{1}=-1.6 \mathrm{GeV}^{2}$.

Above $t_{\text {in }}$, we use a smooth phase $\delta(t)$, which approaches $\pi$ asymptotically - in fact, the results are independent of the phase above $t_{\text {in }}$.

From figure 1, we observe that our best constraints are obtained by including space-like data in addition to the phase and modulus information. Following this, we obtain:

$$
\begin{aligned}
& 3.75 \mathrm{GeV}^{-4} \lesssim c \lesssim 3.98 \mathrm{GeV}^{-4}, \\
& 9.91 \mathrm{GeV}^{-6} \lesssim d \lesssim 10.45 \mathrm{GeV}^{-6} .
\end{aligned}
$$

Comparing with previous determinations of $c$ and $d$, we find that our constraints are in general agreement with those in the literature (see ref. [2] for details).

With regard to complex zeros, we find that the addition of space-like data enlarges the domain where zeros are excluded. In figure 2, we show the domain in the complex plane where zeros are excluded using a space-like datum.

\section{Conclusions}

In this paper, we have obtained stringent bounds on the Taylor coefficients of the pion electromagnetic form factor by exploiting analyticity and unitarity techniques and using various theoretical and experimental inputs. Our results are compared with earlier work and we find that they are in general agreement with some of them. Using the same technique, we have also presented a systematic analysis of zeros of the pion electromagnetic form factor. The knowledge of the zeros is useful for different phenomenological analyses. Our results, which show that zeros are excluded from a rather large region at low energies give support to certain representations (which assume that zeros are absent) and also confirm theoretical expectations based on chiral perturbation theory and more general physical arguments. 


\section{B Ananthanarayan, Irinel Caprini and I Sentitemsu Imsong}

\section{References}

[1] Jefferson Lab Collaboration: G M Huber et al, Phys. Rev. C78, 045203 (2008), arXiv: 0809.3052 [nucl-ex]

[2] B Ananthanarayan, I Caprini and I S Imsong, Phys. Rev. D83, 096002 (2011), arXiv:1102.3299 [hep-ph]

[3] M Davier, A Hoecker, B Malaescu and Z Zhang, Eur. Phys. J. C71, 1 (2011), arXiv:1010.4180 [hep-ph]

[4] R Kaminski, J R Pelaez and F J Yndurain, Phys. Rev. D77, 054015 (2008), arXiv:0710.1150 [hep-ph] 